\title{
LA APLICACIÓN DE ANÁLISIS ARQUEOTECTÓNICOS EN LA ARQUITECTURA DOMÉSTICA EMIRAL DEL TOLMO DE MINATEDA (HELLÍN, ALBACETE)*
}

\author{
APPLICATION OF ARCHITECTONIC ANALYSIS IN DOMESTIC ISLAMIC ARCHITECTURE IN THE TOLMO \\ THE MINATEDA (HELLÍN, ALBACETE)
}

\author{
VÍCTOR CAÑAVATE CASTEJÓN \\ Universidad de Alicante
}

El estudio del conjunto edilicio de época emiral en el Tolmo de Minateda constituye uno de los escasos ejemplos de desarrollo del fenómeno arquitectónico doméstico altomedieval, bien sea en un ámbito general o, como intentaremos exponer en este texto, a una escala microespacial. Durante las últimas campañas de excavación, y en parte gracias a la secuencia estratigráfica, se ha podido evidenciar un proceso evolutivo en el paisaje construido que, en el caso del Tolmo, se ve truncado por el abandono del propio yacimiento. Esta evolución del entramado urbano ha permitido observar cómo los diferentes espacios habitacionales documentados se desarrollan hasta alcanzar una complejidad que en cierta medida justificaría, de una parte, la aparición de un nuevo patrón de asentamiento y, de otra, la descentralización de las actividades que se realizaban en el interior de estos espacios.

En este sentido, el análisis del espacio doméstico a una escala «micro», y su centralidad como argumento, responde a una doble problemática: el desarrollo arquitectónico de los diferentes espacios habitacionales, y la posibilidad de establecer interpretaciones sobre las funciones que se realizarían en cada uno de ellos. En un intento de abordar ambas cuestiones abogamos por el uso de varios análisis que, bajo el marco teórico de la arqueotectura ${ }^{1}$, intentan dar un enfoque arqueológico a la información obtenida a partir de los diferentes elementos arquitectónicos, al tiempo que permiten establecer hipótesis de trabajo siguiendo los resultados obtenidos. Dichos análisis abordan los espacios habitacionales como resultado de una acción eminentemente social; permiten estudiar sus diferentes elementos como partes de un conjunto previamente

\footnotetext{
* Las investigaciones se realizan dentro del proyecto HUM 2006-09874, del Ministerio de Educación y Ciencia.

1. Sobre el desarrollo de este marco teórico se deben ver los trabajos del Laboratorio de Arqueoloxía del Instituto de Estudios Galegos Padre Sarmiento (CSIC-Xunta de Galicia), resumido en parte en J. A. Quirós, 2007.
}

establecido, desglosándolas para luego obtener datos que ayuden a entender los modelos arquitectónicos y su interrelación con otras variables culturales y sociales (concepto familiar, jerarquías, vida doméstica y pública, modos de vida, etc.).

\section{LA METODOLOGÍA ARQUEOTECTÓNI- CA Y SU APLICACIÓN EN CONTEXTOS ALTOMEDIEVALES}

Sin entrar en presupuestos teóricos excesivamente espinosos para la comprensión del tema que nos ocupa, intentaremos presentar la base metodológica que nos ha servido para plantear una serie de interrogantes que, en cierta medida, empezamos a vislumbrar durante el desarrollo de nuestra memoria de licenciatura. Ahora bien, estas cuestiones atienden a los resultados de una serie de analíticas que empezamos a aplicar tras la documentación de las diferentes estructuras exhumadas en el yacimiento del Tolmo, centro de nuestro estudio.

Dentro del desarrollo de las investigaciones científicas llevadas a cabo en el yacimiento del Tolmo de Minateda, se pretende dar un enfoque práctico a una serie de procedimientos de análisis que ya venían siendo utilizados en la Arqueología del Paisaje por el equipo investigador del Laboratorio de Arqueoloxía $(\mathrm{CSIC}-\mathrm{XuGa})^{2}$, que viene desarrollando el estudio de los espacios construidos a partir del marco teórico definido como arqueotectura.

Con el concepto arqueotectura se intenta abordar el registro arquitectónico desde el punto de vista arqueológico. Constituye una vía de estudio que lejos de pretender ser concluyente, permite abordar los problemas

2. Debemos hacer constar la deuda contraída en el presente trabajo con las diferentes publicaciones sobre los planteamientos teóricos y sus aplicaciones prácticas en varios yacimientos por parte del Laboratorio de Arqueoloxía del Instituto de Estudios Galegos Padre Sarmiento (CSIC-Xunta de Galicia). 
planteados en un espacio construido desde un punto de vista arqueológico (Mañana, Blanco y Ayán, 2002, 24). Bajo su definición se agrupan diversos protocolos de actuación cuyos objetivos se centran en la interpretación del registro arquitectónico; de esta forma, cada una de las estancias que conforma una vivienda no se debe interpretar como una acción independiente, sino que debe ser observado desde una óptica procesual, donde cada construcción se encuentra formando parte de un producto generado por un fenómeno cultural concreto. En cierta medida se trata del estudio del espacio construido, con carácter doméstico y residencial, desde un enfoque que combina la documentación de las diferentes estructuras que conforman ese espacio con el uso de herramientas específicas; unos análisis que permitan interrelacionar los diferentes elementos que componen el espacio construido (Ayán, 2003,17-18).

La metodología analítica empleada para el estudio del carácter funcional y doméstico de los niveles emirales del yacimiento del Tolmo contempla diversos procedimientos de análisis:

- Análisis secuencial. Partiendo de la secuencia estratigráfica, establecemos un área de trabajo susceptible de ser analizado por su carácter unitario. A partir de la definición de espacio habitacional (en adelante E.H.), por el cual entendemos todas aquellas estructuras de habitación que dan sentido a una vivienda, registramos el orden secuencial y cronológico de cada uno de los elementos que los componen (muros, umbrales, bancos, hogares, tinajeras) y, en el caso que sea posible, su relación estratigráfica (activa y pasiva) con los demás.

- Análisis formal. Mediante este análisis se pueden llegar a entrever los principios organizadores y jerarquizadores de un E.H. (Ayán, 2003, 19), así como intuir la funcionalidad de los diferentes ambientes o estancias que lo componen. En este sentido se pretende observar la configuración del espacio atendiendo a su articulación interna y su disposición en relación al espacio exterior, jugando por otra parte, con la relación espacial y con la organización espacial (Ching, 1995, 178-227; Mañana, Blanco y Ayán, 2002, 33-35).

- Análisis de circulación. Tras establecer un orden cronológico para las diferentes construcciones que componen el E.H., y señalar su orden formal, el análisis de circulación pretende identificar el orden perceptivo de cada estancia o ambiente a partir del movimiento de los espacios (Ching, 1995, 228), evidenciar su capacidad de integración con el resto e intentar averiguar qué zonas cobran preeminencia visual. El resultado de la aplicación de esta herramienta viene determinado por el control de ciertos elementos constructivos que influyen y dirigen la circulación (Mañana, 2003, 178), y que en nuestro caso podrían responder a pasillos y umbrales escalonados.

Esta herramienta materializa en diagramas la forma de acceso, la configuración del recorrido y las relaciones del recorrido con el espacio al que se va acceder (Ching, 1995, 228-277 y Mañana, Blanco y Ayán, 2002, 37).
- Análisis de permeabilidad o gamma. Consiste en el estudio de la segregación de los espacios de una casa o poblado a través de la relación de contigüidad de los espacios. Mediante gráficas de permeabilidad, esta herramienta permite cuantificar el grado de acceso y permeabilidad de las diferentes estancias o ambientes que conforman un espacio habitacional (Mañana, 2003, 178), al tiempo que valora el nexo que tienen entre ellos estableciendo grados de dependencia, así como de la propia relación entre el exterior y el E.H., y éste con los diferentes ambientes o estancias que lo componen.

Este tipo de análisis, que fue desarrollado por Hillier y Hanson (1984), permite determinar el grado de jerarquía de los diferentes espacios a partir de la permeabilidad o facilidad de acceso que estos tienen desde el exterior, al tiempo que establece un orden de profundidad que se superpone al significado social -y funcional- de cada espacio. De esta forma, el análisis gamma facilitaría la comprensión y la «conectividad» de aquellos espacios ubicados en zonas aparentemente «apartados» del resto (alcobas, zonas de máxima privacidad), dotados con un mayor grado de segregación; y los que tienden a abrirse a los espacios públicos (patios privados, espacios susceptibles de ser interpretados como artesanales, almacenes, etc.), que presentan menor grado de segregación y por tanto más integrados al circuito interno del E.H.

Asimismo, las gráficas de permeabilidad generadas pueden determinar distintos tipos de relación entre los espacios que las componen (distribuida, no distribuida, simétrica, asimétrica, arbusto poco profundo, arbusto profundo, anillo poco profundo y anillo profundo). A partir de ahí se calcula la profundidad media y el índice de permeabilidad, indicando más o menos segregación, y por tanto, más o menos dificultades de comunicación (Hillier y Hanson, 1984, 143 y ss.)

- Análisis de visibilidad. Mediante el uso de este análisis se pretende valorar el grado de privacidad de cada uno de los ambientes o estancias que componen un E.H., siempre y cuando constituyan una construcción cerrada. Como el análisis gamma, supone una herramienta de gran valor a la hora de establecer jerarquías entre espacios, identificando los grados de privacidad y la carga simbólica de que están dotados; de otra parte facilita la comprensión de los motivos sociales, económicos y culturales para que una familia o comunidad construyan, vivan y modifiquen esos espacios. En este sentido, el análisis de visibilidad permite señalar que un espacio, cuanto más visible, mayor carga pública conlleva, y cuanto menos visible, mayor es su privacidad.

La aplicación de este análisis depende de la percepción que un individuo tiene del interior de un espacio desde un punto de vista concreto, y que normalmente se realiza desde el vano de acceso a cada estancia o ambiente. Ahora bien el grado de visibilidad dependerá de varios factores, condicionando la separación teórica entre las zonas más visibles y las menos visibles; 


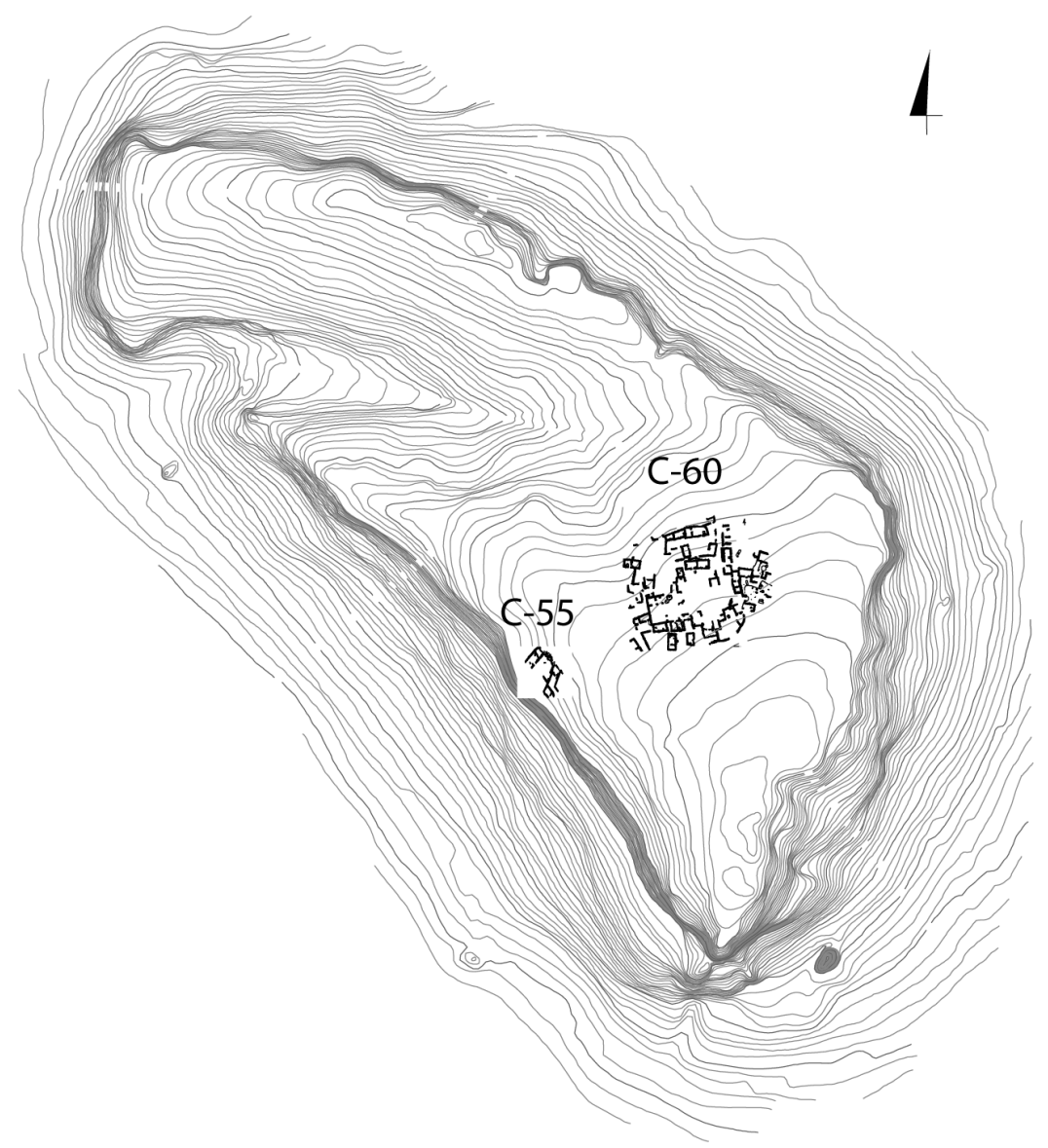

Figura 1: Planta del Tolmo de Minateda con los cortes de excavación a los que haremos referencia.

por un lado el ancho del vano y su ubicación (centro, lateral o esquinado) y, por otro, el grosor de los muros que conforman el espacio (Ayán, 2003, 19).

La aplicación práctica de estas herramientas al ámbito urbano de época altomedieval, en lo que conocemos, cuenta con escasos ejemplos; por ello la investigación del entorno doméstico y de la vida cotidiana desde el marco del análisis microespacial está aún por desarrollar. Sin embargo, creemos que el uso de esta metodología debe dar resultados positivos en tanto se tengan en cuenta las diversas características de las sociedades a las que corresponden dichos espacios habitacionales. El trasfondo sociocultural de las nuevas poblaciones que se van asentando en el yacimiento del Tolmo configura en cierta medida la génesis de un cambio estructural y arquitectónico, donde la formación social islámica rompe con el sustrato indígena desde el punto de vista urbanístico ${ }^{3}$, aportando por otra parte, una ideología propia que subyace en la arquitectura de las viviendas de época emiral documentadas en el yacimiento.

3. Sobre la continuidad de la ciudad en época emiral y su proceso de islamización, ver: M. Acién, 2001; S. Gutiérrez, 2007; S. Gutiérrez, e.p.
Ahora bien, el desarrollo de las herramientas arqueotectónicas debe entenderse no tanto desde el punto de vista individual de cada una de las construcciones a las que se aplican, sino que debe ponerse en común con los demás. Cada construcción, sea estancia o habitación o ambiente, debe entenderse como un objeto con capacidad de relacionarse con el medio en el que está construido y con el resto de objetos que le rodean.

\section{ESTUDIO ARQUEOTECTÓNICO EN LOS ESPA- CIOS HABITACIONALES 1 Y 6 (C-60), Y EN EL COMPLEJO EDILICIO DEL CORTE 55}

La documentación de una extensa trama urbana emiral en varios cortes del yacimiento nos ha servido para aplicar sobre varios conjuntos edilicios las herramientas expuestas anteriormente -en concreto en los denominados Corte 55 y Corte 60 (Figs. 1 y 2)-, resultando clarificadores a la hora de explicar el proceso de cambio y ruptura con el sustrato previo. Ahora bien, es cierto que algunos factores urbanísticos sugieren una cierta continuidad con la edilicia visigoda; la documentación de un conjunto aparentemente doméstico en la zona intramuros de la puerta invita a pensar en una cierta similitud formal entre las construcciones 


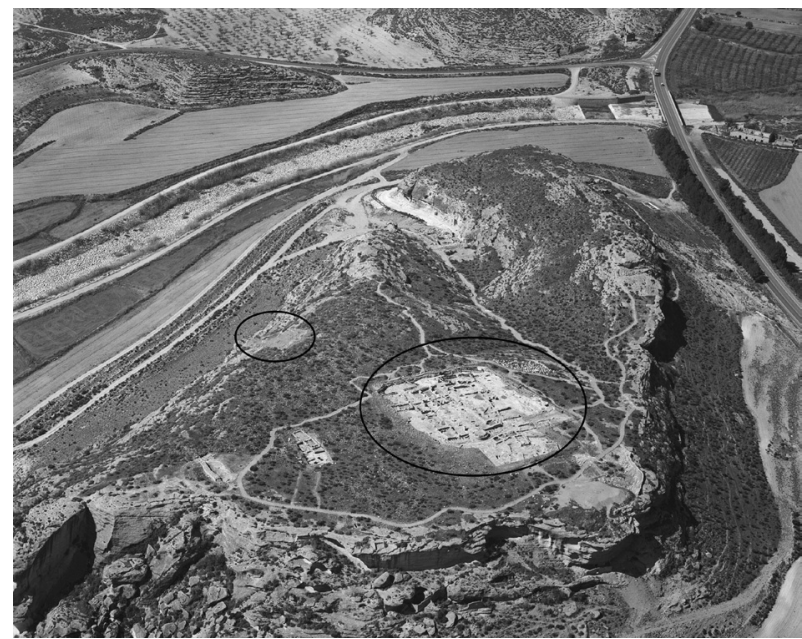

Figura 2: Vista aérea del yacimiento con los cortes señalados.

visigodas e islámicas: construcciones aparentemente simples, de planta cuadrangular o rectangular, dispuestas en torno a un espacio abierto que actúa a modo de patio semiprivado, e inconexas entre sí (Gutiérrez, e.p.). Se trata, pues, del modelo conformado por estructuras monocelulares, caracterizadas por poseer únicamente una estancia y por la simplicidad constructiva (Bazzana, 1992, 164- 168), y dotadas de al menos un hogar, elemento indispensable que dada su doble acepción -lugar en el que se habita y dispositivo para el fuego (Cañavate, e.p.)- permite definir los espacios destinados a la vivienda.

En este sentido, y siguiendo los análisis secuencial y formal, observamos cómo las nuevas estructuras, que podemos vincular a los inicios de la islamización del yacimiento, comienzan a erigirse sobre las ruinas de la edilicia visigoda. La documentación de diferentes construcciones en la plataforma superior del cerro -sobre todo en el denominado Corte 60-, parece evidenciar un proceso ininterrumpido de ocupación que sugiere un cambio sustancial con la planta de época visigoda. Tal es así que si bien es cierto que buena parte del edificio con carácter litúrgico se emplea como cantera de extracción de materiales para las nuevas edificaciones, parte del palatium continúa en pie al menos durante la germinación del nuevo entramado emiral, transformándose algunas de sus dependencias en pequeñas viviendas que conviven con las primeras edificaciones ex поио (Fig. 3).

No obstante, el desarrollo del nuevo paisaje arquitectónico parece reflejar por un lado el progresivo abandono de la gran mayoría de las construcciones anteriores, quedando su reempleo en un mero testimonio de lo que fue el complejo visigodo ${ }^{4}$, y por otro,

4. Contamos con algunos ejemplos de estancias emirales de nueva construcción que utilizan parte de los muros visigodos, aún en pie, como uno de los cierres; sin embargo, son casos que podemos considerar excepcionales, más teniendo
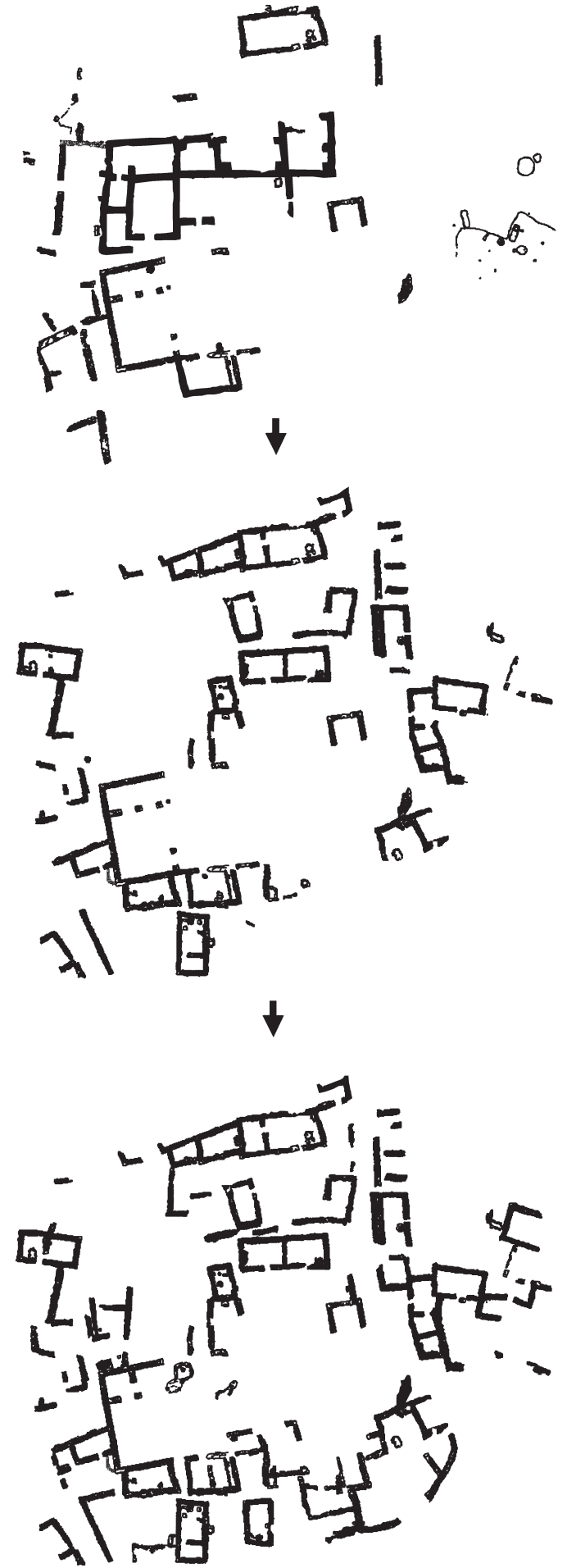

Figura 3: Evolución de la trama urbana emiral en el Corte 60.

la proliferación de diversas construcciones, aparentemente aisladas o adosadas, pero que tienden a estar articuladas por espacios abiertos de gran superficie

en cuenta que la gran mayoría de los muros conservados de época visigoda aparecieron amortizados bien por las nuevas edificaciones, bien por los paquetes de derrumbe y colmatación previos al desarrollo constructivo del barrio emiral. 


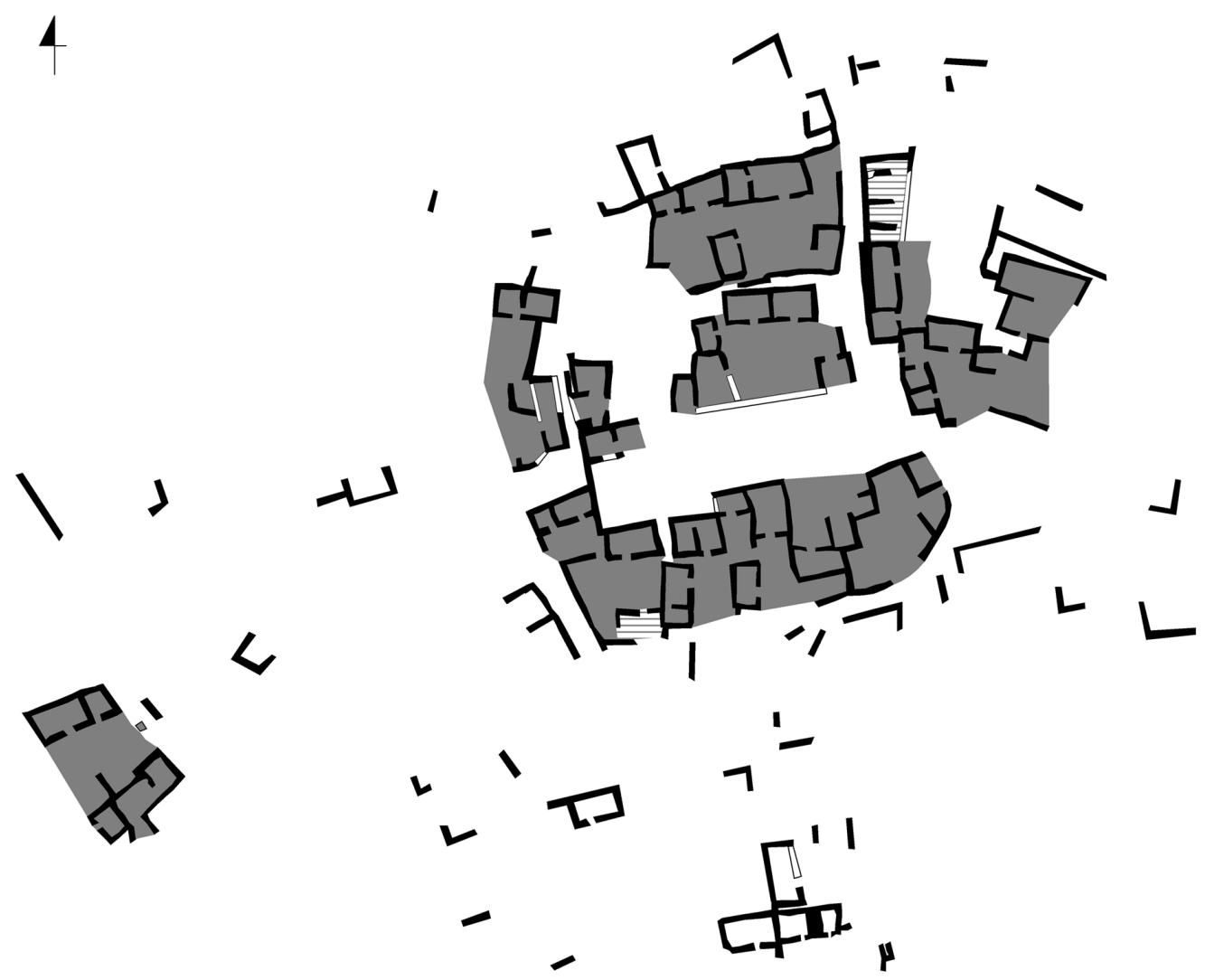

Figura 4: Mapa que representa la separación entre espacios abiertos (blanco) y privados (gris).

con accesos más o menos restringidos desde las zonas públicas. Surge así una retícula de complejos de estancias casi siempre monocelulares, vertebrados por patios probablemente de ámbito semiprivado, y separados por calles y explanadas de carácter público ${ }^{5}$ que permiten el acceso a través de un sistema de accesos estrechos (pasillos acodados o azucates) definidos por las propias unidades de habitación (Fig. 4).

Por otra parte, en el Corte 55 evidenciamos una serie de construcciones que estratigráficamente podemos vincular a los dos últimos estadios de ocupación del yacimiento sin poder definir, en cambio, una progresiva transformación arquitectónica. Se trata de dos complejos arquitectónicos con estancias monocelulares de planta rectangular, uno de ellos dispuesto en torno a un patio o espacio articulador que permitiría el acceso desde el exterior y a la única construcción correspondiente a una fase de ocupación anterior: un aljibe rupestre con poceta y mortero hidráulico que

\footnotetext{
5. Debemos señalar que una de estas explanadas es en realidad el solar de la basílica visigoda, en la que se concibieron dos hornos, por lo que es más que probable que se transformara en un área abierta con un claro carácter artesanal.
}

durante la época emiral se reemplea en un centro de almacenaje o silo ${ }^{6}$.

Pese al cambio en el panorama constructivo y a la aparente ausencia de criterios urbanizadores, el mapa axial (Fig. 5) muestra un eje norte-sur y otro este-oeste que articulan el ordenamiento interno, amén de definir cada uno de los citados complejos a modo de «manzanas» que se van adaptando a las condiciones del terreno. De otra parte parece existir un planteamiento urbanístico con calles, algunas de las cuales están fosilizando las vías de comunicación, al menos, desde época visigoda ${ }^{7}$, pero que ahora tienden a complicar su

6. Desconocemos el momento exacto en que la estructura rupestre dejó de emplearse como aljibe; sin embargo, la existencia en los paquetes de colmatación de abundante material cerámico adscrito al primer Horizonte, evidencian la interrupción de este uso antes de la edificación de las estructuras de habitación documentadas en el exterior. Para el estudio de los horizontes cerámicos altomedievales del Tolmo, ver S. Gutiérrez, B. Gamo, y V. Amorós, 2003.

7. Una de estas vías responde a un camino rupestre con sendas carriladas que bordeaba el ábside de la basílica, y que fue ocupada en parte por un cementerio ad sanctos. De igual modo parece ocurrir en las cercanías del denominado Corte 55, donde registramos un tramo de vía rupestre con marcas de rodadas que, con una orientación norte-sur, permitiría el acceso a los complejos edilicios documentados en el citado corte. 


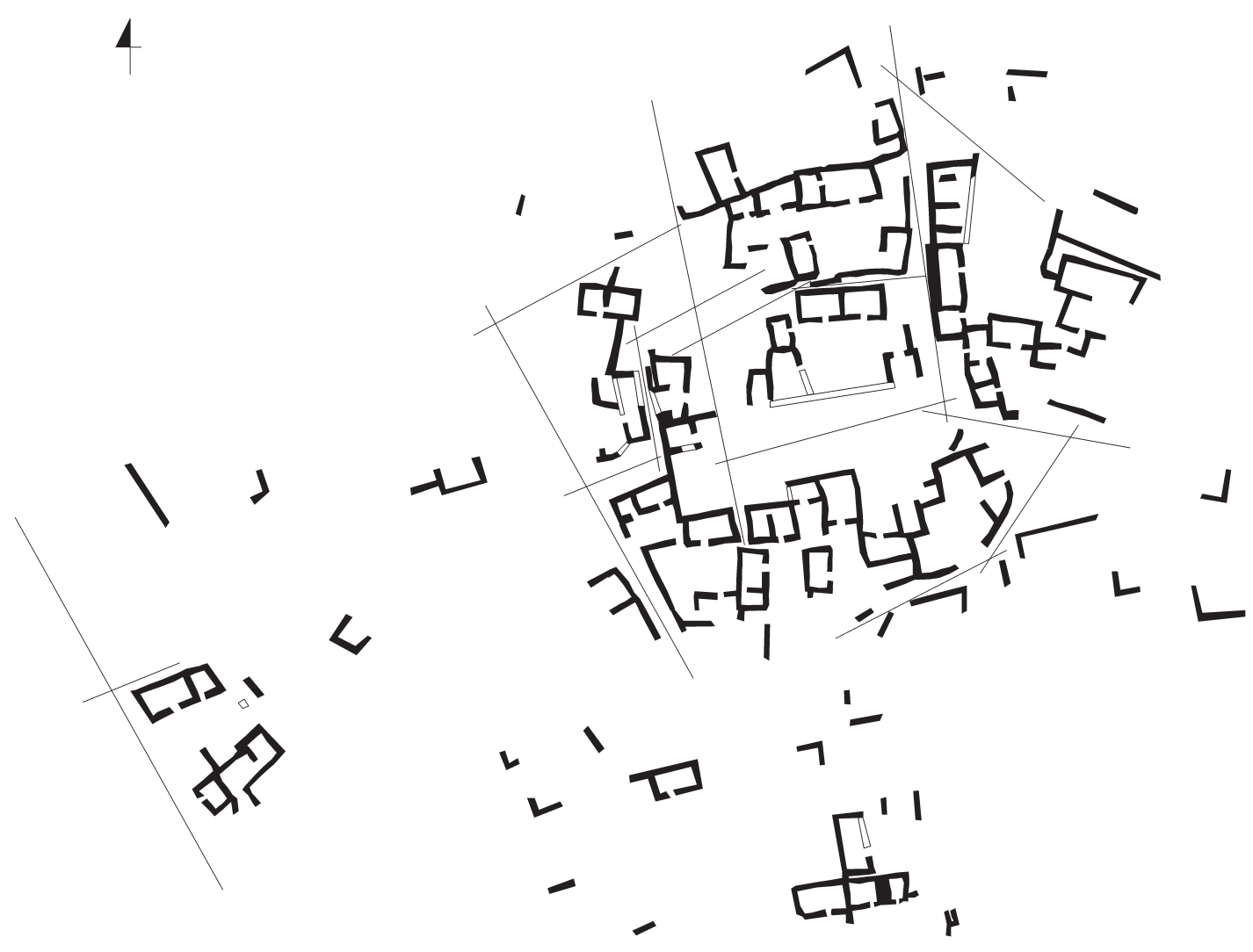

Figura 5: Mapa axial de los Cortes 60 y 55.

recorrido siguiendo unas pautas constructivas preestablecidas, como puede ser la edificación de espacios habitacionales a oriente de lo que fue el complejo episcopal de época visigoda.

Los análisis de circulación, visibilidad y gamma de los espacios habitacionales completan a escala microespacial los grados de acceso, visualización y permeabilidad de los mismos, atendiendo a una jerarquización y división interna de cada uno de los complejos. En este sentido, creemos conveniente exponer tres ejemplos de aplicación de las herramientas utilizadas: Los EE.HH. 1 y 6 del Corte 60 y el complejo doméstico del Corte 55.

Los tres responden al mismo modelo formal; estancias de planta rectangular, articuladas en torno a un patio bien delimitado por tabiques ex profeso o por las edificaciones pertenecientes a otros EE.HH. Se trata de edificaciones que se disponen aprovechando los espacios edificables, bien sea sobre la superficie de la propia roca, sobre las ruinas de las construcciones visigodas, o sobre una superficie previamente acondicionada que crea un sistema de aterrazamiento con pendiente hacia el norte o hacia los límites del propio cerro. Ahora bien, esta adaptación al terreno queda en cierta medida condicionada por la planificación previa que parece inferir en la gran mayoría de los EE.HH. documentados; tal es así que los complejos aparecen bien delimitados por los espacios públicos que los circundan.
Sus muros son de mampostería irregular, que en ocasiones reemplea material constructivo romano o visigodo, con un sistema de jambaje resuelto en la mayoría de los casos con grandes losas de piedra arenisca. Desconocemos en cambio el sistema de cubrición de estas estructuras, sin que aún se pueda afirmar con rotundidad si se trataba de cubiertas planas, atestiguadas en la Rábita de Guardamar (Gutiérrez, e.p.; Azuar et alii, 1991, 61) o con cierta inclinación ${ }^{8}$. Destaca, sin embargo, la diferencia de cota entre los niveles de frecuentación de las estancias y de los patios, siendo frecuente la existencia de umbrales escalonados que salven la altura de piso entre el «interior» y el «exterior» de los E.H.

Del mismo modo documentamos diversas estructuras de combustión ubicados en diferentes puntos de los tres E.H., dominando los situados en el interior de las estancias, junto a los muros, esquinas o vanos de acceso; y frecuentemente acompañados de otras estructuras que dan sentido a usos y funcionalidades concretas (tinajeras, vasares o asientos). Son este tipo de construcciones las que constituyen unas estructuras primordiales a la hora de establecer interpretaciones

8. Evidentemente las condiciones meteorológicas deben influir en el tipo de cubierta utilizada, no sólo en el tipo de materiales empleados sino en el grado de inclinación de las propias cubiertas (Bazzana, 1992, 164). 


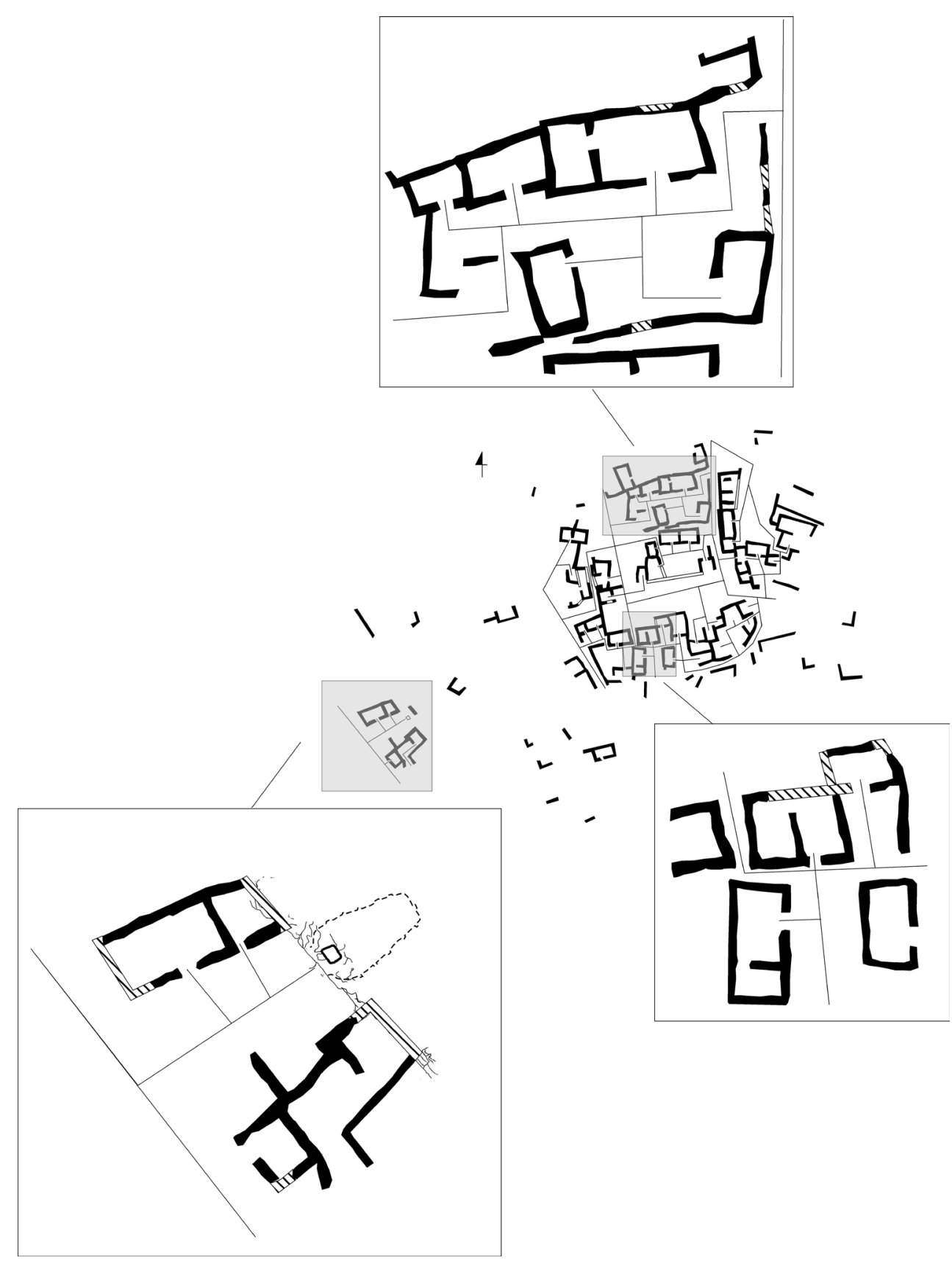

Figura 6: Representación gráfica de la circulación entre los diferentes EE.HH. Ampliados los tres ejemplos estudiados.

globales en una unidad de habitación, ya que justifican unos usos muy concretos, como la cocción de alimentos, iluminación del espacio y el poder calorífico (Cañavate, e.p.).

En otro sentido, la aplicación del análisis de circulación (Fig. 6) y su plasmación gráfica muestra cómo en los tres complejos existe una organización espacial distribuida, es decir, únicamente existe una vía de acceso y ésta queda supeditada al control del patio, verdadero epicentro y eje articulador de la vivienda. La presencia de tabiques adosados a algunas de las estancias (como es el caso del E.H. 1) y el uso de las traseras de otros complejos (como en el E.H. 2 y en el complejo del Corte 55), denotan la existencia de filtros reguladores de acceso, resaltan la impermeabilidad con respecto a los espacios públicos y en consecuencia, realzan la restricción preestablecida que dota a los tres complejos de una mayor carga de privacidad.

Asimismo las herramientas de permeabilidad y visibilidad (Fig. 7) nos permiten esbozar cuál es el patrón de asentamiento de estos EE.HH., extensible por lo demás al resto de complejos exhumados en los diferentes cortes del yacimiento, y explicable por la llegada de un nuevo modelo social de articulación espacial fosilizado en el entramado urbanístico que empieza a 

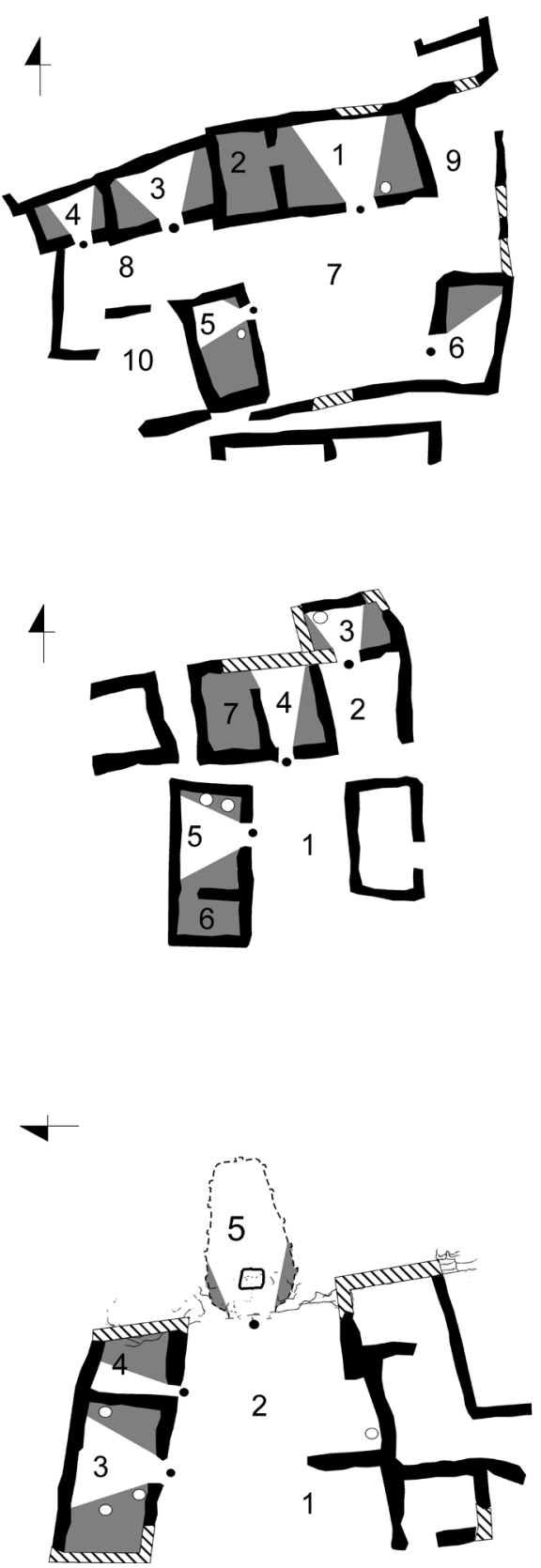

E.H. 1

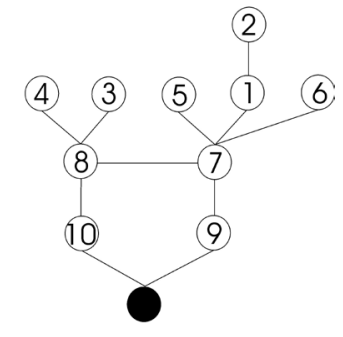

E.H. 6

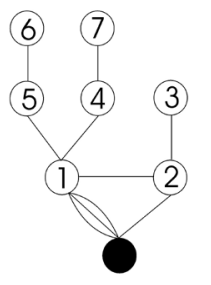

Corte 55

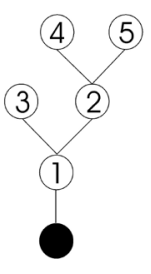

Figura 7: Representación gráfica del análisis de visibilidad y diagrama de permeabilidad en los conjuntos estudiados.

desarrollarse en el Tolmo ${ }^{9}$. Este nuevo patrón de asentamiento queda reflejado por la aparición de variaciones importantes en las diferentes construcciones. En

9. Debemos recordar que los niveles emirales del Tolmo constituyen la última fase de ocupación del yacimiento. En este sentido, asistimos a la fosilización del entramado urbano precalifal, entendiéndolo desde una doble perspectiva: el despoblado del Tolmo constituye un campo único de experimentación y documentación de los primeros niveles islámicos en la Península que, sin embargo, interrumpe el desarrollo del nuevo modelo social de articulación espacial. primer lugar, empezamos a documentar una subdivisión interna de las propias estancias, normalmente con la aparición de tabiques medianeros que crean ambientes bien diferenciados y separados. Esta compartimentación del espacio supone dotar a uno de los dos ambientes de una mayor carga de privacidad, al tiempo que quedan expuestos a una menor visibilidad, un acceso más restringido, y en consecuencia, se erigen como los espacios menos permeables. La aplicación del análisis de permeabilidad relativa (Fig. 8), muestra que son las estancias separadas por tabiques las que tienden a estar más segregadas y menos integradas 
con respecto al resto de los espacios que conforman el conjunto habitacional ${ }^{10}$. Son estos espacios los que carecen de cualquier tipo de estructura doméstica interna (hogares, vasares, bancos o tinajeras), o de ajuar; son los espacios de menores dimensiones, por lo que asignarles una función que vaya más allá de la propia alcoba o área de descanso de la vivienda parece cuanto más inverosímil. Esta división interna del espacio nos permite pensar que los EE.HH. documentados se encuentran en la antesala de los modelos homogéneos basados en la casa con patio, zaguán en recodo, crujías con alcobas, letrina y espacio independiente para cocinar (Gutiérrez, e.p.), que son típicos de la arquitectura doméstica islámica ${ }^{11}$; al tiempo que parecen alejarse de los tipos urbanos indígenas, caracterizados por estructuras unicelulares, más o menos dispersas.

En segundo lugar, y en relación con el primero, el acceso a cada uno de los diferentes espacios habitacionales tiende a restringirse; se complica la circulación directa desde los espacios públicos mediante la construcción de elementos arquitectónicos que limitan y dotan de privacidad a los espacios domésticos. Del mismo modo, la complejidad urbanística supone que algunos cierres de otros espacios habitacionales permiten mediatizar los accesos.

En tercer lugar, la ubicación de las estructuras vinculadas a las labores domésticas (hogares, tinajeras, bancos, etc.) tienden a construirse en las zonas de menor visibilidad, dotándolas con un mayor carga de privacidad; por lo que se crea una compartimentación no física dentro de una misma estancia que a priori podría carecer de ella. En este sentido, empiezan a aparecer tabiques medianeros que delimitan ambientes dentro de la propia construcción, menos permeables desde el exterior, más privados, y en consecuencia, dentro de la jerarquía espacial, más importantes.

Estas transformaciones permiten que se empiece a percibir un proceso de discontinuidad a doble nivel; por un lado la ruptura con los patrones de asentamiento propios de las sociedades indígenas, propiciado por el desarrollo del concepto racional de habitabilidad de las sociedades islámicas; y por otro, la aparición de un nuevo modelo social vinculado a la cultura islámica,

10. Para calcular la «permeabilidad relativa» (P.R.) desde cualquier punto, se calcula la «profundidad media» (P.M.) del complejo asignando un valor de profundidad a cada estancia o ambiente de acuerdo con cuántos espacios estén alejados del origen, sumando estos valores y dividiéndolos por el número total de estancias que forman el complejo menos uno; de esta forma, la P.M. se calcula mediante la fórmula: $\mathrm{AR}=2(\mathrm{PM}-1) / \mathrm{K}-2$, donde $\mathrm{PM}$ es la «profundidad media» y $\mathrm{K}$ el número de estancias que forman el complejo. El resultado siempre oscilará entre 0 y 1 ; en este sentido, cuanto más cerca de 1 esté indicará más segregación y por tanto más dificultades de comunicación con el resto de estancias.

11. Como es el caso de Bayyâna Pechina, yacimiento urbano con cronología temprana donde se documentaron complejos plenamente islámicos. Ver F. Castillo y R. Martínez, 1990. que supone el surgimiento de nuevas tendencias constructivas, un nuevo modelo de ocupación espacial y un cambio en la adaptación al medio físico. La compartimentación de los espacios habitacionales conlleva la aparición de estancias diferenciables, así como ambientes dentro de una misma estancia, lo cual parece reflejar una diversidad económica en el ámbito doméstico.

Las casas tienden a agruparse, a formar parte de un amplio conglomerado que se organiza en tramas urbanas con identidades propias; ejercen un control propio jerarquizando espacios internos y restringiendo accesos. En definitiva, un nuevo orden que refleja cambios sustanciales.

\author{
Víctor Cañavate Castejón \\ Área de Arqueología \\ Dpto. Prehistoria, Arqueología, Historia Antigua, \\ Filología Griega y Filología Latina \\ Facultad de Filosofía y Letras \\ Universidad de Alicante \\ 03080 Alicante \\ victor.canavate@ua.es
}

\section{BIBLIOGRAFÍA}

ABAD, L., GutiÉrRez, S. y SANZ, R., 1998: El Tolmo de Minateda (Hellín, Albacete): una historia de 3.500 años, Toledo.

ACIÉN, M., 2001: «La formación del tejido urbano en alAndalus», en PASSINI J. (Coord.): La ciudad medieval: de la casa al tejido urbano, 11-32, Universidad de Castilla-La Mancha.

Aicha-AzzizA, A. y Fentress, E., 1990: «Sétif: evolution d'un quartier», La casa hispanomusulmana. Aportaciones de la arqueología, 163-176, Granada.

Aicha-AzZIZA, A. y Fentress, E., 2003: «Arquitectura como tecnología de construcción de la realidad social», Arqueología de la Arquitectura, 2, 17-24.

AYÁn, X. M., 2001: Arqueotectura 2: La vivienda castreña. Propuesta de reconstrucción en el castro de Elvira, Traballos en Arqueoloxía da Paisaxe, 23, Santiago de Compostela.

Azuar, R., Beviá, M., Borrego, M. y Salanova, R., 1991: «La Rábita de Guardamar (Alicante): su arquitectura», Cuadernos de Madînat al-Zahrâ, 2, 55-83.

BAZZANA, A., 1992: Maisons d'al-Andalus. Habitat médiéval et structures du peuplement dans l'Espagne orientale, Madrid.

BAZZANA, A., 1993: «Matériaux, techniques et modes de construction: quelques réflexions à propos de l'habitat villageois dans al-Andalus», Castrum 6, Maisons et espaces domestiques dans le monde Méditerranéen aun Moyen Âge, 53-74, Rome-Madrid.

CAstillo, F., y Martínez, R. 1990: «La vivienda hispanomusulmana en Bayyâna Pechina», La casa hispanomusulmana. Aportaciones de la Arqueología, 111-128, Granada.

CHING, F. 1995. Arquitectura: forma, espacio y orden, México. 
CRIADO, F., 1993. «Visibilidad e interpretación del registro arqueológico» Trabajos de Prehistoria, 50b, 39-56, Madrid.

GUTIÉRREZ, S., 1993: «De la ciuitas a la madîna: destrucción y formación de la ciudad en el sureste de al-Andalus. El debate arqueológico», IV Congreso de Arqueología Medieval Española (Alicante, 1993), 12-35, Alicante.

GUTIÉRREZ, S., 2000: «El espacio doméstico altomedieval del Tolmo de Minateda (Hellín, Albacete), entre el ámbito urbano y el rural», Castrum 6, Maisons et espaces domestiques dans le monde Méditerranéen aun Moyen Âge, 151-64, Rome-Madrid.

GuTIÉRREZ, S., 2002: «De espacio religioso a espacio profano: transformación del área urbana de la basílica del Tolmo de Minateda (Hellín, Albacete) en barrio islámico», II Congreso de Historia de Albacete (Albacete, noviembre del 2000).

GUTIÉRREZ, S., 2007: «la islamización de Tudmir: balance y perspectivas», Villes et campagnes de Tarraconaise et d'al-Andalus (VI'-XI siècles): la transition, 275-318, Université de Toulouse-Le Mirail.

GUTIÉRREZ, S., e.p.: «Madinat Iyyuh y la destrucción del espacio urbano en la Alta Edad Media», Castrum 8, El Castillo y la Ciudad. Espacios y redes (ss. VI-XIII).
Gutiérrez, S., GAMO, B. y AMORÓs, V., 2003: «Los contextos cerámicos altomedievales del Tolmo de Minateda y la cerámica altomedieval en el sudeste de la Península Ibérica», Cerámicas tardorromanas y altomedievales en la Península Ibérica. Ruptura y continuidad (II Simposio Internacional de Mérida, 2001), Anejos de Archivo Español de Arqueología, XXIII, 119-68.

HiLlier, B. y HANSON, J, 1984: The social logic of space, Cambridge.

IZQUIERDO, R., 1994: Ciudad hispanomusulmana «Vascos», Navalmoralejo (Toledo). Campañas 19831988, Toledo.

KENT, S., 1990: Domestic Architecture and the use of space. An interdisciplinary cross-cultural study, Cambridge.

MAÑANA, P., 2003: «Arquitectura como percepción», Arqueología de la Arquitectura, 2, 177-183.

MañanA, P., Blanco, R. y AYÁn, X. M., 2002: Arqueotectura 1: bases teórico-metodológicas para una arqueología de la Arquitectura, Traballos en Arqueoloxía da Paisaxe, 23, Santiago de Compostela.

QUIRÓS, J. A., 2007: «La arqueología de la arquitectura y la arqueología medieval (por qué hacer historia a partir del registro arquitectónico de época medieval)», Tendencias actuales en arqueología medieval, 23-57, Murcia. 